\title{
Integral equation technique for scatterers with mesoscopic insertions: Application to a carbon nanotube
}

\author{
M. V. Shuba, ${ }^{1}$ A. V. Melnikov, ${ }^{1}$ P. P. Kuzhir, ${ }^{1}$ S. A. Maksimenko, ${ }^{1}$ G. Y. Slepyan, ${ }^{2}$ A. Boag, ${ }^{2}$ A. Mosca Conte, ${ }^{3}$ \\ O. Pulci, ${ }^{4,5}$ and S. Bellucci ${ }^{6}$ \\ ${ }^{1}$ Institute for Nuclear Problems, Belarus State University, Bobruiskaya 11, 220050 Minsk, Belarus \\ ${ }^{2}$ School of Electrical Engineering, Tel Aviv University, Tel Aviv 69978, Israel \\ ${ }^{3}$ ISMN, Consiglio Nazionale delle Ricerche, Via Salaria Km 29, 30000015 Monterotondo Stazione (Roma), Italy \\ ${ }^{4}$ Department of Physics, University of Rome Tor Vergata, and INFN, Via della Ricerca Scientifica 1, I-00133 Rome, Italy \\ ${ }^{5}$ CNR-ISM, Via del Fosso del Cavaliere 100, I-00133 Rome, Italy \\ ${ }^{6}$ Frascati National Laboratory, National Institute of Nuclear Physics, E. Fermi 40, 00044 Frascati, Italy
}

(Received 19 April 2017; revised manuscript received 21 August 2017; published 8 November 2017)

\begin{abstract}
We present the electromagnetic scattering theory for a finite-length nanowire with an embedded mesoscopic object. The theory is based on a synthesis of the integral equation technique of classical electrodynamics and the quantum transport formalism. We formulate Hallén-type integral equations, where the canonical integral operators from wire antenna theory are combined with special terms responsible for the mesoscopic structure. The theory is applied to calculate the polarizability of a finite-length single-walled carbon nanotube (CNT) with a short low-conductive section (LCS) in the microwave and subterahertz ranges. The LCS is modeled as a multichannel two-electrode mesoscopic system. The effective resistive sheet impedance boundary conditions for the scattered field are applied on the CNT surface. It is shown that the imaginary part of the polarizability spectrum has three peaks. Two of them are in the terahertz range, while the third is in the gigahertz range. The polarizability spectrum of the CNT with many LCSs has only one gigahertz peak, which shifts to low frequencies as the number of LCSs increases. The physical nature of these peaks is explained, and potential applications of nanoantennas are proposed.
\end{abstract}

DOI: 10.1103/PhysRevB.96.205414

\section{INTRODUCTION}

Over the past two decades, the methods of electrical engineering have been evolving toward a self-consistent description of mesoscopic structures with a feature size larger than the atomic distance but smaller than the characteristic length at which quantum correlations already appear [1]. The recent progress in nanoscience has paved the way for experimental implementation of different nanosized objects, such as quantum dots [2], molecular and quantum contacts $[3,4]$, carbon nanotubes [5], and graphene [4].

Electron transport in coherent conductors with a size comparable to, or less than, the inelastic scattering length has a number of specific features. The most important feature is the nonlocality of electron transport between two reservoirs [1]. This process can be described by using a well-known particle scattering theory that considers electrons scattering by a potential barrier from an initial to a final state. The transition from one state to another is described by the scattering matrix [1]. The first impressive result in the quantum transport theory based on this approach is the famous Landauer formula for a quasi-one-dimensional coherent mesoscopic conductor [6].

Due to the recent development of nanotechnologies, mesoscopic structure changed from a research subject into an element of nanoelectronic devices. Electron reservoirs for coherent conductors can act as interconnects, waveguides, antennas, and rectennas for the electromagnetic (EM) field. For example, mesoscopic structures such as quantum contacts or quantum dots pave the way to efficiently controlling and loading nanoantennas in the infrared and optical frequency ranges [7-9].

The trend toward the integration of nanophotonics with mesoscopic physics requires us to build a bridge between classical electrodynamics and quantum transport theory [10-12]. Electrodynamics of nanostructures is based, to a large degree, on the principles and methods of classical electrodynamics. For example, the integral equation technique for light scattered by different types of nanowires (e.g., carbon nanotubes $[13,14]$, noble metal wires [8], etc.) originates in classical antenna theory [15]. Integral equations for surface currents in nanowires are in general similar to the Hallén integral equations for macroscopic wire antennas [15], though their specific forms are slightly different due to a difference in the constitutive relations of nanostructures and macroscopic bulk media; the latter are usually modeled as dielectrics or perfect conductors.

The subject of this paper is the electromagnetic scattering theory for a quantum nanowire (or ribbon) with embedded mesoscopic structure. The key idea consists of combining the integral equation technique of classical electrodynamics and a formalism of the high-frequency quantum transport theory. The mesoscopic structure is modeled with the help of the scattering matrix approach [1]. Its interaction with the EM field is described by the alternating-current (ac) voltage across the structure and the total ac current through it. The nanowire is modeled with the help of the effective boundary conditions for the EM field. Its interaction with the EM field is described by the density of the surface current induced by the total (external plus scattered) field [16]. The current density and the voltage between two reservoirs are unknowns and should be found self-consistently from the boundary-value problem for the EM field.

The framework of the analysis is a modified integral equation in which the Hallén-like integral operator appears simultaneously with the special terms responsible for the 
mesoscopic structure. This equation is valid for an arbitrary type of coherent conductors and quantum wires, whose physical origin manifests itself only in the values of the electron transmission coefficient and conductance, respectively. Such parameters appear in the integral equations as a priori given, and they should be found separately via numerical modeling or experiment.

Our approach will be illustrated by analyzing the problem of the scattering of an EM wave by a finite-length carbon nanotube (CNT) with a short low-conductive section (LCS) in the microwave and terahertz ranges. The CNT will be considered as a nanowire, whereas the LCS will be modeled as a mesoscopic conductor. The LCS could be (i) a nanosized defective section of the CNT [17], (ii) a junction constructed between tubes of different chiralities [18], or (iii) a molecular bridge between tips of the CNTs [19]. Hereafter, the CNT with the LCS will be referred to as d-CNT.

If the LCS is a molecular bridge between the tips of the two CNTs, the LCS conductance might be controlled in different ways, including optical, chemical, electrical, and mechanical methods:

(i) The conductance of some molecules can be switched chemically. For example, the molecular conductance of oligoaniline diamine 4 changes by nearly an order of magnitude following the variation in $\mathrm{pH}$ level of the environment [19].

(ii) The conductance of some photochromic molecules is sensitive to visible and ultraviolet light. It has been demonstrated experimentally that diarylethene molecules can be converted from a conjugated "on" state to a cross-conjugated "off" state upon illumination in the visible range [20,21]. The reverse process is possible with ultraviolet light.

(iii) The molecular conductivity can be controlled by a transverse electric field. This is actively used in a single molecular transistor [22-24].

(iv) The conductance of the molecular bridge depends strongly on its geometrical configuration. For instance, the conductance of a molecule $\mathrm{C}_{60}$ placed between $(5,5)$ carbon nanotubes is determined by the distance between nanotubes and the orientation of the fullerene. It can be tuned by rotating one of the CNTs around its axis [25].

(v) The conductance of some molecules can also be varied by their mechanical stretching [26].

Electromagnetic parameters of the CNTs have been investigated intensively in the past two decades [13,14,27-38]. Their unique electronic properties and folding geometry made CNTs into promising components for electronic and electromagnetic applications in the microwave and terahertz ranges. Carbon nanotubes are currently proposed as elements for different integrated circuits and electromagnetic devices, such as transmission lines [28,38], interconnects [33], and nanoantennas $[13,14,30]$. Composite materials comprising the low content of the CNTs as inclusions demonstrate good shielding properties in gigahertz [39] and terahertz [40] regimes.

Due to the high kinetic inductance of a single-walled CNT, the strongly slowed-down surface wave can propagate along its axis $[28,38]$. The excitation of the standing surface wave by a plane wave causes antenna (or localized plasmon) resonance in the polarizability spectrum of an individual finite-length CNT [14]. A terahertz conductivity peak of a CNT film [27,29,34] is proven experimentally to be due to the localized plasmon resonance in the CNTs [41].

In the frequency range below the localized plasmon resonance $(<1 \mathrm{THz})$, a quasistatic interaction between the CNT and the incident electromagnetic wave occurs. In this case, the strong depolarizing field leads to the screening effect- the total axial field is as much as $10-10^{3}$ times smaller than the axial component of the incident field $[35,36]$. Moreover, due to the polarization effect, a high surface charge density is concentrated near the CNT edges. These charges induce a radial electric field that is 10-30 times higher than an axial component of the incident field, resulting in the near-field enhancement effect $[35,42,43]$.

Though the electromagnetic parameters of perfect CNTs have been well established $[14,31,36,42]$, there are no theoretical or experimental studies of the d-CNT. In the present paper, we propose a systematic study of the problem of electromagnetic wave scattering by a single-walled d-CNT. We shall show that the d-CNT has qualitatively different terahertz and microwave polarizability spectra as compared to those for the CNT without the LCS. The electron transmission coefficients for some particular LCSs were modeled based on first-principles approaches (a plane-wave formalism within density-functional-theory calculations [44]).

The paper is organized as follows. In Sec. II, we present a brief review of the Landauer model of a mesoscopic structure driven by the ac field, we formulate the boundaryvalue problem for the mesoscopic structure embedded into a nanowire, and we develop the general integral-equation-based technique for its solution. The calculated conductance of some particular LCSs in CNT is discussed in Sec. III. The application to the problem of plane waves scattering by a finite-length $\mathrm{d}-\mathrm{CNT}$ in the microwave and terahertz ranges is analyzed and discussed in Sec. IV. The polarizability spectra of the d-CNTs with many LCSs are presented and discussed in Sec. V. The possibilities of tuning the LCS conductivity and possible applications of the d-CNTs in nanoantennas are discussed in Sec. VI. The conclusions are drawn in Sec. VII.

\section{THEORETICAL FRAMEWORK}

Some examples of electromagnetic systems with mesoscopic structures are shown in Fig. 1. Each system consists of a nanoantenna with a gap bridged by a nanojunction. The latter is a mesoscopic object connecting two terminals of the nanoantenna. The coupling between the terminals is dictated by the electron tunneling through the gap. The description of tunneling is based on the concept of quantum transport [1,45-47].

The famous Landauer model was originally proposed for the case of dc voltage produced by a battery and applied to a pair of electrodes (leads) separated by a nanoscale junction. The battery acts as a reservoir of the electrons, i.e., it can supply and receive an arbitrary amount of electrons and energy without changing its internal state. Each lead is connected to a reservoir. The electrons were prepared in the distant past in the reservoirs far away from the junction. They move toward the junction from the leads scattering elastically on the junction potential. After that they move away without further scattering. The energy required to extract an electron from one reservoir 
(a)

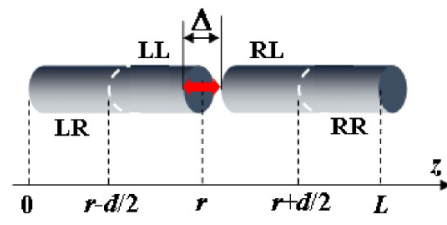

(c)

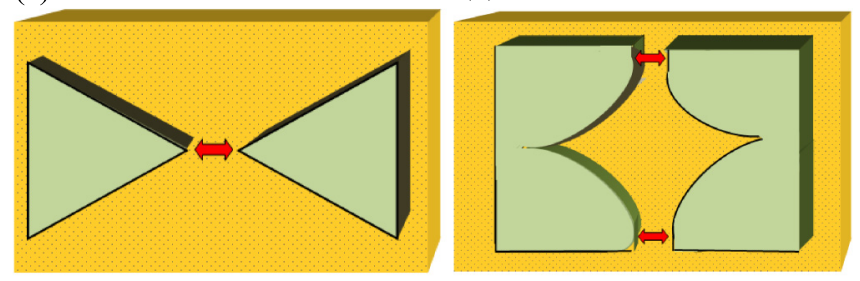

FIG. 1. Examples of the electrodynamic systems with mesoscopic structures. (a) Nanowire decomposed following the LandauerBüttiker concept of quantum transport. LR, LL, RL, and RR denote left-hand reservoir, left-hand lead, right-hand lead, and right-hand reservoir, respectively. (b) Circular nanoloop, (c) nanoribbon, and (d) Vivaldi antenna [48] placed on the optically transparent background. Double red arrows indicate electron tunneling junctions.

and bring it into the system must be different for different reservoirs. Such a difference is interpreted $[1,45,46]$ as the difference in electrochemical potentials associated with the left- and right-hand reservoirs.

This physical picture cannot be directly applied to the case of nanoantennas due to the following reasons.

(i) A nanoantenna is exposed to an externally impressed ac field with angular frequency $\omega$. In this case the Landauer scenario of quantum transport does not require the assumption of different chemical potentials of the right- and left-hand reservoirs [47].

(ii) The voltage between the antenna terminals is created by the total field, which should be determined self-consistently from the boundary-value problem for Maxwell equations and the quantum transport model. Thus, this problem should take into account the electromagnetic interaction between the nanojunction and the reservoir.

\section{A. A model of a quantum wire with an imbedded mesoscopic structure}

As an example, for further consideration we choose a quantum wire with a number of conductive channels $N_{c}$. Let the wire be aligned parallel to the $z$ axis of a cylindrical coordinate system and be exposed to an externally impressed field with $z$ component $E_{z}^{\mathrm{ex}}(z)=E_{z}^{(0)}(z) \exp (-i \omega t)$. Hereafter, we will use the International System of units.

Let the quantum wire have electron scattering obstacles localized in a short region $z \in(r-\Delta / 2, r+\Delta / 2)$ [see Fig. 1(a)]. We define $T_{i}$ and $R_{i}\left(i=1,2, \ldots, N_{c}\right)$ as the total transmission and reflection coefficients of this region for an electron into channel $i$. Let us choose a section of length $d(d>$ $\Delta)$ with the scattering obstacles in the center. We shall refer to such a section as an LCS. The length $d$ can be associated with a distance between the voltage probes in the scheme of the LCS conductance measurements. In general, this distance is different for "two-" and "four-terminal" schemes [46]. The length $d$ should be much smaller than the electron mean free path $\lambda_{\mathrm{mfp}}$ in the wire (i.e., $d \ll \lambda_{\mathrm{mfp}}$ ) providing electron elastic scattering within the LCS. Since the typical value of $\lambda_{\text {mfp }}$ is much smaller than an electromagnetic wavelength $\lambda$, the condition $d \ll \lambda$ is satisfied automatically, allowing for a quasistatic description of the electromagnetic field within the LCS.

The voltage drop across the LCS can be found from the total field $E_{z}^{\text {tot }}(z)$ within the LCS in a quasistatic approximation as follows:

$$
V_{d}=\int_{r-d / 2}^{r+d / 2} E_{z}^{\mathrm{tot}}(z) d z
$$

The voltage $V_{d}$ should be related self-consistently to the EM field scattered by the remaining part of the system.

The description of the field and charge distribution within the LCS is a complex quantum-mechanical task, which should be solved separately for each particular configuration of the scattering obstacles. In our formulation, we will proceed with the LCS as a section with an effective conductance $G_{d}$, which can be determined via the quantum transport formalism. We use two basic assumptions in our model: (i) a weak-field approximation $\left(\omega \hbar \gg e V_{d}\right)$, and (ii) the low-frequency regime (see below). Then, the current $I_{d}$ through the LCS and the voltage $V_{d}$ are connected linearly $[1,47,49]$,

$$
I_{d}=G_{d}(\omega) V_{d}
$$

To calculate $G_{d}$, one can apply different models of quantum transport. Here we mention two of them.

In the framework of the Landauer-Büttiker formalism for a potential barrier with scattering-free leads connected to two reservoirs [49] [see Fig. 1(a)], $G_{d}$ can be found from "twoterminal" many-channel conductance formulas [49]. This formalism is quite general; it also allows (i) an investigation of the rectification effect and shot noises in the system [49], (ii) generalization of the theory for the case of a wire with a double barrier at the resonant tunneling [49], and (iii) development of the model for the system with embedded mesoscopic structure having more than two terminals [50,51]. It should be noticed that the formula for "two-terminal" conductance yields unreasonable finite conductance in the absence of electron scattering, i.e., at $T_{i}=1, i=1,2, \ldots, N_{c}$.

In the framework of the low-frequency Landauer formalism developed by Kamenev and Kohn in Ref. [47] for a barrier imbedded in the quantum wire, $G_{d}$ can be calculated using the "four-terminal" conductance formula.

Though both models (Refs. [49] and [47]) yield the same value of $G_{d}$ for a weakly transmitting $\operatorname{LCS}\left(T_{i} \ll 1\right.$, $\left.i=1,2, \ldots, N_{c}\right)$, their outcomes are rather different for a weakly reflecting $\operatorname{LCS}\left(R_{i} \ll 1, i=1,2, \ldots, N_{c}\right)$. The reason originates from the difference of the problem definitions (see details in [47]).

Since our task and the boundary-value problem for the LCS are close to those considered by Kamenev and Kohn, we shall further apply their "four-terminal" conductance formulas [47] derived for the barrier with disordered leads in the cases of a 
weakly transmitting LCS,

$$
G_{d}(\omega) \approx \frac{e^{2}}{\pi \hbar} \sum_{i=1}^{N_{c}} T_{i}
$$

and a weakly reflecting LCS,

$$
G_{d}(\omega) \approx \frac{e^{2}}{\pi \hbar} \frac{\left[\sum_{i=1}^{N_{c}} v_{i}\right]}{\sum_{i=1}^{N_{c}} v_{i}^{2} R_{i}},
$$

where $\hbar$ is the reduced Planck constant, $e$ is the electron charge, and $v_{i}$ is the electron velocity in channel $i$. For the particular case of equal velocity channels and arbitrary $T_{i}$ and $R_{i}$, one can apply [47]

$$
G_{d}(\omega)=\frac{e^{2}}{\pi \hbar} \frac{N_{c} \sum_{i=1}^{N_{c}} T_{i}}{\sum_{i=1}^{N_{c}} R_{i}} .
$$

Formulas (3)-(5) take into account the spin degeneracy. They are correct when (i) the frequency is small, $E_{F} \gg \omega \hbar$, allowing us to neglect the difference in the velocities of scattered and incident electronic states [52], and (ii) the values $T_{i}$ and $R_{i}$ are slowly varying functions on the scale of both temperature $T$ and frequency $\omega$. The chemical potential is supposed to be the same on both sides of the LCS.

Since $V_{d}$ is a voltage in the "four-terminal" measurement scheme, then following Ref. [47], the value of $d$ in (1) should be taken to be equal to $\Delta+2 \kappa^{-1}$, where $\kappa^{-1}$ is the Thomas-Fermi screening length along the wire.

\section{B. Boundary-value problem for a d-CNT}

Here, we propose the electromagnetic boundary-value problem formulation to describe the electrodynamics of metallic d-CNT. Let an isolated d-CNT of length $L$ and cross-sectional radius $a$, aligned parallel to the $z$ axis of a Cartesian coordinate system [see Fig. 1(a)], be located in a host medium with permittivity $\varepsilon$ (host relative permittivity is $\left.\varepsilon_{h}=\varepsilon / \varepsilon_{0}, \varepsilon_{0}=8.85 \times 10^{-12} \mathrm{~F} / \mathrm{m}\right)$. The CNT occupies the region $z \in(0, L)$, and the LCS with length $d$ is located in the interval $z \in(r-d / 2, r+d / 2) ; 0<r<L$.

Let the d-CNT be exposed to an externally impressed field with a $z$-component $E_{z}^{\mathrm{ex}}(z)=E_{z}^{(0)}(z) \exp (-i \omega t)$. This field induces the surface current density $\mathbf{j}(\mathbf{r})$ in the d-CNT, which reradiates the scattered field.

The electron scattering by the obstacles located in the center of the LCS causes charge accumulation near them. Due to the poor screening of the long-range Coulomb interaction in CNTs [53], the charge density decays slowly (nonexponentially) with the distance from the scattering obstacles along the $z$ axis. In spite of the long-range decay (of several tens of the CNT radius), the main decrease in the charge density occurs in the short interval_ "screening length" $\kappa^{-1}$ —comparable with CNT diameter [53]. Therefore, it is reasonable to assume the LCS length is in the range $d \in(\Delta+4 a, \Delta+10 a)$.

In the terahertz and subterahertz regime, only intraband transitions of $\pi$ electrons with unchanged transverse quasimomentum are allowed in the CNT [54]. As those transitions contribute to the axial conductivity, but not to the transverse conductivity, only the azimuthally symmetric electric current densities can be excited in the CNTs [31]. So, we can set $\mathbf{j}(\mathbf{r})=j(z) \mathbf{e}_{z}$, where $\mathbf{e}_{z}$ is the unit vector along the CNT axis. (a)

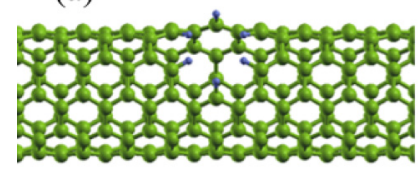

(c)

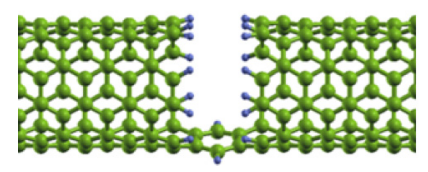

(b)

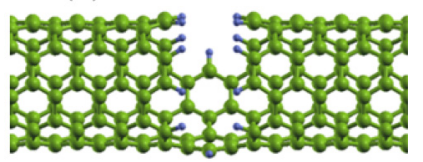

(d)
FIG. 2. Defects of (5,5) armchair CNT with (a) 6, (b) 18, (c) 24, and (d) 60 carbon atoms extracted. Carbon dangling bonds are hydrogen-passivated.

The Landauer concept of quantum transport deals only with a total current $I_{d}$, whereas an electromagnetic task requires the introduction of a spatially distributed current density within the LCS. The realistic current density is complex in the azimuthally asymmetric scattering region (see Fig. 2). To simplify the electromagnetic task and match it with the model of mesoscopic structure, we assume the current to be uniformly distributed on the surface of the LCS, which is modeled as a hollow cylinder of radius $a$ and length $d$. The linear relation between voltage across the LCS, $V_{d}$ [see Eq. (1)], and current through the LCS, $I_{d}$, is determined by (2) where the effective conductance $G_{d}$ is given by (3) or (4). Then the effective current density within the LCS is

$$
j(z)=j_{d}=\frac{I_{d}}{2 \pi a}=\frac{1}{2 \pi a} G_{d} V_{d}, \quad z \in(r-d / 2, r+d / 2) .
$$

Thereby, we ignore in our model the real geometry of the LCS. We shall show below that the polarizability of the d-CNT does not depend on the value $d$, but only on the value of the LCS effective conductance $G_{d}$, thus justifying the validity of our simplification.

Since the surface current on the CNT surface outside the LCS in the local approximation satisfies Ohm's law, then using (6) we can present the surface current density in the d-CNT as follows:

$$
j(z)=\left\{\begin{array}{l}
\sigma_{c n} E_{z}^{\mathrm{tot}}(z), z \in(0, r-d / 2) \cup(r+d / 2, L), \\
j_{d}, z \in(r-d / 2, r+d / 2),
\end{array}\right.
$$

where the total axial field $E_{z}^{\text {tot }}$ on the CNT surface can be found as a sum of axial components of incident $E_{z}^{(0)}$ and scattered $E_{z}^{\mathrm{sc}}$ fields,

$$
E_{z}^{\mathrm{tot}}(z)=E_{z}^{(0)}+E_{z}^{\mathrm{sc}},
$$

while $\sigma_{c n}$ in (7) is the axial surface conductivity of the CNT; for a small radius metallic CNT $(<2 \mathrm{~nm})$ [28],

$$
\sigma_{c n}=\frac{2 i e^{2} v_{F}}{\pi^{2} \hbar a(\omega+i v)},
$$

where $v_{F}$ is the Fermi velocity for a CNT, $v_{F} \simeq 10^{6} \mathrm{~m} / \mathrm{c} ; v$ is the relaxation frequency; $v=\tau^{-1}$, where $\tau$ is the electron relaxation time. 
Let us note that $G_{d}$ in (3)-(5) is purely real. In general, the dynamic admittance $Y_{d}(\omega)$ should be complex. At small frequencies, it can be expanded to first order in frequency as follows [51,52]: $Y_{d}(\omega)=G_{d}(0)+i B_{d} \omega$, where $B_{d}$ is a constant value.

For a weakly transmitting LCS, the admittance $Y_{d}$ has a capacitive imaginary part $[47,55]$ taking into account the electric charges accumulated within the LCS due to the electron scattering effect, $B_{d}=-C<0$, where $C$ is a capacitance of the LCS. For sufficiently small LCS length $d$, these charges are much smaller than those accumulated outside of the LCS in its locality. Hence, in our further consideration, we shall neglect the charges accumulated within the LCS, omitting the capacitive part of the admittance, i.e., assuming $B_{d}=0$.

For weakly reflecting LCS, the admittance has an inductive imaginary part, $B_{d}>0$, which is important in the highfrequency range. The positive imaginary part of the CNT admittance appears due to the inertia of electrons moving in the ac electric field. If this field slightly changes during the period of the electron relaxation time, then the electron motion is very similar to that in a dc field. Therefore, in the range $f \ll$ $v /(2 \pi)$, the imaginary part of both $\sigma_{c n}$ and $Y_{d}$ is much smaller than the real one, and it can be omitted. In our calculations, we shall use an electron relaxation time of $\tau=50 \mathrm{fs}[\nu /(2 \pi) \approx 3$ THz] [34]. Then formula $Y(\omega)=G_{d}(0)$ describe the LCS admittance with high accuracy in the range $f \in(0,0.5) \mathrm{THz}$. Since we shall consider further only the case of weakly transmitting LCS providing a small inductive imaginary part of $Y_{d}$, we shall also apply and presume the validity of the equality $Y_{d}(\omega)=G_{d}(0)$ in the range $f \in(0.5,10) \mathrm{THz}$.

Electromagnetic properties of the CNT in our model are described by the effective resistive sheet impedance boundary conditions for the total electric, $\mathbf{E}^{\text {tot }}$, and magnetic, $\mathbf{H}^{\text {tot}}$, fields. In a general form, they are written as follows [28]:

$$
\begin{gathered}
\mathbf{n} \times\left(\mathbf{E}_{+}^{\mathrm{tot}}-\mathbf{E}_{-}^{\mathrm{tot}}\right)=0 \\
\mathbf{n} \times \mathbf{E}^{\mathrm{tot}}=-\hat{Z}\left[\mathbf{n} \times \mathbf{n} \times\left(\mathbf{H}_{+}^{\mathrm{tot}}-\mathbf{H}_{-}^{\mathrm{tot}}\right)\right]=0,
\end{gathered}
$$

where subscripts \pm indicate different sides of the surface, $\mathbf{n}$ is the local outward unit vector normal to the surface, and $\hat{Z}$ is the tensor of the sheet impedance. Those effective boundary conditions were first adapted for CNTs [28] and thereafter for graphene [56].

The electromagnetic field scattered by the d-CNT can be described in terms of the electric Hertz potential $\Pi(\mathbf{r})=$ $\Pi(\rho, z) \mathbf{e}_{z}$, which satisfies the Helmholtz equation:

$$
\left(\nabla^{2}+k^{2}\right) \Pi=0
$$

where $k=\sqrt{\varepsilon_{h}} \omega / c$ is the wave number, and $c$ is the speed of light in vacuum. The only nonzero components of the scattered electromagnetic fields are as follows:

$$
E_{\rho}^{\mathrm{sc}}=\frac{\partial^{2} \Pi}{\partial z \partial \rho}, \quad E_{z}^{\mathrm{sc}}=\left(\frac{\partial^{2}}{\partial z^{2}}+k^{2}\right) \Pi, \quad H_{\phi}^{\mathrm{sc}}=i \varepsilon \omega \frac{\partial \Pi}{\partial \rho} .
$$

The boundary conditions for Eq. (12) for the d-CNT can be written as follows [28]:

$$
\begin{array}{r}
\left.\frac{\partial \Pi}{\partial \rho}\right|_{\rho=a+0}-\left.\frac{\partial \Pi}{\partial \rho}\right|_{\rho=a-0}=\frac{j(z)}{i \omega \varepsilon}, \quad z \in(0, L), \\
\left.\frac{\partial \Pi}{\partial \rho}\right|_{\rho=a+0}=\left.\frac{\partial \Pi}{\partial \rho}\right|_{\rho=a-0}, \quad z \in(-\infty, 0) \cup(L,+\infty), \\
\left.\Pi\right|_{\rho=a+0}=\left.\Pi\right|_{\rho=a-0}, \quad-\infty<z<+\infty .
\end{array}
$$

The total axial field $E_{z}^{\text {tot }}$ on the CNT surface can be found from (8) subjected to (13),

$$
E_{z}^{\mathrm{tot}}(a, z)=E_{z}^{(0)}(z)+\left(\frac{\partial^{2}}{\partial z^{2}}+k^{2}\right) \Pi(a, z) .
$$

To obtain the unique solution of the boundary-value problem for the $\mathrm{CNT}$, the boundary conditions must be supplemented by radiation conditions [57] as well as by edge conditions. The latter express the absence of concentrated charges on the edges $z=0, L$ of the tube, i.e.,

$$
j(0)=j(L)=0 .
$$

The formulated boundary-value problem can easily be generalized for the d-CNT with many LCSs when the distance between any adjacent LCSs is larger than the electron coherence length. In this case, the strong dephasing processes occur in the region between adjacent LCSs providing uncorrelated electron scattering by different LCSs.

\section{Hallén-type equation for a d-CNT}

The solution of Helmholtz equation (12) with boundary conditions (14)-(16) and edge conditions (18), taking into account (6), may be represented as a single-layer potential,

$$
\begin{aligned}
\Pi(\rho, z)= & \frac{i a}{4 \pi \varepsilon \omega}\left(\int_{0}^{r-d / 2} j\left(z^{\prime}\right) G\left(\rho, z-z^{\prime}\right) d z^{\prime}\right. \\
& +j_{d} \int_{r-d / 2}^{r+d / 2} G\left(\rho, z-z^{\prime}\right) d z^{\prime} \\
& \left.+\int_{r+d / 2}^{L} j\left(z^{\prime}\right) G\left(\rho, z-z^{\prime}\right) d z^{\prime}\right),
\end{aligned}
$$

where $j(z)$ and $j_{d}$ are the unknown surface current densities to be found, and

$$
G(\rho, z)=\int_{0}^{2 \pi} \frac{\exp \left(i k \sqrt{a^{2}+\rho^{2}-2 \rho a \cos (\varphi)+z^{2}}\right)}{\sqrt{a^{2}+\rho^{2}-2 \rho a \cos (\varphi)+z^{2}}} d \varphi .
$$

The potential $\Pi(a, z)$ can be expressed from (17) as follows:

$$
\Pi(a, z)=\frac{1}{2 i k} \int_{0}^{L} E_{z}^{\mathrm{tot}}(a, z) e^{i k\left|z-z^{\prime}\right|} d z^{\prime}-\frac{1}{2 i k} \Phi(z),
$$

where

$$
\Phi(z)=\int_{0}^{L} E_{z}^{(0)}(z) e^{i k\left|z-z^{\prime}\right|} d z^{\prime}+C e^{i k z}+D e^{-i k z},
$$

where $C$ and $D$ are unknown constants to be determined from edge conditions (18). 
Let us expand the integral in Eq. (21) as follows:

$$
\int_{0}^{L}\{\cdots\} d z^{\prime}=\int_{0}^{r-d / 2}+\int_{r-d / 2}^{r+d / 2}+\int_{r+d / 2}^{L}\{\cdots\} d z^{\prime} .
$$

Taking into account (1), (2), (6), and the inequality $k d \ll$ $k L \ll 1$, the middle integral on the right side of (23) can be transformed as follows:

$$
\begin{aligned}
& \int_{r-d / 2}^{r+d / 2} E_{z}^{\mathrm{tot}}\left(z^{\prime}, a\right) e^{i k\left|z-z^{\prime}\right|} d z^{\prime} \\
& \simeq e^{i k|z-r|} \int_{r-d / 2}^{r+d / 2} E_{z}^{\mathrm{tot}}\left(z^{\prime}\right) d z^{\prime} \\
& \quad=V_{d} e^{i k|z-r|}=\frac{2 \pi a}{G_{d}} j_{d} e^{i k|z-r|} .
\end{aligned}
$$

In view of (7) and (24), after substitution of (21) into (19) at $\rho=a$, we arrive at the Hallén-type equation for the d-CNT with respect to the current density $j(z)$ and effective current density $j_{d}$,

$$
\begin{aligned}
& \int_{0}^{r-d / 2}+\int_{r+d / 2}^{L}\left[\sigma_{c n}^{-1} e^{i k\left|z-z^{\prime}\right|}+\frac{a k}{2 \pi \varepsilon \omega} G\left(a, z-z^{\prime}\right)\right] \\
& \quad \times j\left(z^{\prime}\right) d z^{\prime}+j_{d} F(z)=\Phi(z),
\end{aligned}
$$

where

$$
F(z)=\frac{2 \pi a}{G_{d}} e^{i k|r-z|}+\frac{k a}{2 \pi \varepsilon \omega} \int_{r-d / 2}^{r+d / 2} G\left(a, z^{\prime}-z\right) d z^{\prime} .
$$

Let us note that the first term in (26) contains $G_{d}$ but not $d$. This means that the surface current density distribution over the d-CNT and, consequently, the d-CNT polarizability does not depend on LCS length $d$, but only on the conductance $G_{d}$. For all the d-CNTs considered in Sec. IV, we proved with numerical calculations that the solution of Eq. (25) practically does not depend on the value of $d$ if it varies from 0.1 to $5 \mathrm{~nm}$ for a constant $G_{d}$. The independence of the electromagnetic parameters of the d-CNT on the LCS length justifies the suggestion that the application of the approach developed for the LCS of cylindrical shape could be also applied for any other LCS of noncylindrical geometry. This is true due to the one-dimensional nature of the electron transport in CNTs.

The integrals in (25) can be handled by a quadrature formula, thereby transforming (25) into a matrix equation. The latter can be solved numerically to find the current density in the d-CNT.

Let us note that Eq. (25) has a solution that is very similar to a solution of the Hallén equation for a CNT with $z$-dependent axial conductivity, i.e., instead of (7) one can assume

$$
j(z)=\sigma(z) E_{z}^{\text {tot }}(z), \quad z \in(0, L),
$$

where

$$
\sigma(z)=\left\{\begin{array}{l}
\sigma_{c n}, z \in(0, r-d / 2) \cup(r+d / 2, L), \\
\frac{G_{d} d}{2 \pi a}, z \in(r-d / 2, r+d / 2) .
\end{array}\right.
$$

For this case, the Hallén equation for the CNT with $z$ dependent conductivity has the form

$$
\int_{0}^{L}\left[\frac{1}{\sigma\left(z^{\prime}\right)} e^{i k\left|z-z^{\prime}\right|}+\frac{a k}{2 \pi \varepsilon \omega} G\left(a, z-z^{\prime}\right)\right] j\left(z^{\prime}\right) d z^{\prime}=\Phi(z) .
$$

Our calculations show that the solutions of Eqs. (25) and (29) coincide within the error of $1 \%$ for all the d-CNTs considered in Sec. IV. Moreover, these equations can be easily generalized to calculate the electromagnetic response of the d-CNT with an arbitrary number of noncorrelated LCSs.

\section{Electromagnetic parameters of a d-CNT}

Once we know the surface current density, we can calculate a polarizability tensor of the d-CNT in the long-wavelength regime $(L \ll \lambda)$. The tensor has only one nonzero element,

$$
\alpha_{z z}=\frac{2 \pi i a}{\omega E_{z}^{(0)}} \int_{0}^{L} j(z) d z .
$$

Hereafter, we shall refer to $\alpha_{z z}$ as CNT polarizability.

The surface charge density on the d-CNT outside the LCS can be found from the continuity equation as

$$
\rho(z)=-\frac{i}{\omega} \frac{\partial j(z)}{\partial z}, \quad z \in(0, r-d / 2) \cup(r+d / 2, L) .
$$

Let us note that both (25) and (29) together with (31) give the correct charge-density distribution $\rho(z)$ outside the LCS. However, the value $\rho(z)$ within the LCS depends on its intrinsic atomic structure, and, consequently, it cannot be described by the presented model.

As we considered elastic electron scattering by the LCS, the electromagnetic energy cannot be dissipated within the LCS. However, the power $P_{d}$ gained by electrons passing through the LCS is dissipated within a distance of the order of the electronic mean free path from the LCS [58]. It can be obtained as

$$
P_{d}=2 \pi^{2} a^{2} G_{d}^{-1}\left|j_{d}\right|^{2} .
$$

The power $P_{c n}$ dissipated in the d-CNT by electrons that did not pass the LCS can be calculated as follows [42]:

$$
P_{c n}=\pi a \operatorname{Re}\left(\sigma_{c n}^{-1}\right)\left[\int_{0}^{r-d / 2}+\int_{r+d / 2}^{L}|j(z)|^{2} d z\right] .
$$

Then the total dissipated power in the d-CNT is a sum of $P_{c n}$ and $P_{d}$

\section{THE CONDUCTANCE OF SOME PARTICULAR LCSs}

To demonstrate which types of defects might be associated with the LCS in the d-CNT, as an example we calculated the conductance of some particular defects in a metallic armchair $(5,5)$ CNT (see Fig. 2). As shown in Fig. 2, the LCSs were constructed by extraction of $6,18,24$, and 60 carbon atoms. In practice, similar defects could be obtained by voltage pulses [59].

Extraction of some carbon atoms leads to the emergence of dangling-bond defects in the CNTs. They are chemically reactive and tend to adsorb gas molecules such as $\mathrm{CO}_{2}$ and $\mathrm{O}_{2}$ from the air [60]. To make the d-CNT chemically stable in air, we passivated all the dangling bonds by hydrogen atoms. To get mechanically stable atomic configurations, we carried out structural optimization of the LCS with the PWSCF code of the QUANTUM ESPRESSO package, which uses a plane-wave formalism based on ground-state density-functional-theory calculations [44]. 
TABLE I. The conductance $G_{d}(0)$ and the ratio $N_{\sigma}=$ $2 \pi a \sigma_{c n}(0) /\left(G_{d} d\right)$ for the defects presented in Fig. 2.

\begin{tabular}{lcr}
\hline \hline Number of extracted C atoms & $G_{d}\left(e^{2} \pi^{-1} \hbar^{-1}\right)$ & $N_{\sigma}$ \\
\hline 6 & 1.06 & 33 \\
18 & 0.16 & 220 \\
24 & 0.015 & 2320 \\
60 & 0.0042 & 8300 \\
\hline \hline
\end{tabular}

The $(5,5)$ armchair CNT has two conductive channels near the Fermi level [61]. To calculate electron transmission probability $T_{i}$ for the $i$-channel $(i=1,2)$, we used a rigorous first-principles method [62] (the Choi-Ihm method) based on the scattering theory and pseudopotentials, which are implemented within the PWCOND part of the QUANTUM ESPRESSO package [44]. The calculations were performed using the PW91 [63] exchange and correlation functional. The convergence with the number of plane waves and with the number of $k$-points used to sample the Brillouin zone was carefully checked. We used an energy cutoff of $35 \mathrm{Ry}$, and a set of $12 k$-points.

LCS conductance $G_{d}(0)$ has been calculated according to (8) assuming that the velocity of electrons moving in two channels is equal to $v_{F}$. The LCS conductance and the value $N_{\sigma}=2 \pi a \sigma_{c n}(0) /\left(G_{d} d\right)$ at $d=5 \mathrm{~nm}, a=0.34 \mathrm{~nm}$, and $\tau=50 \mathrm{fs}$ are collected in Table I for all the defects presented in Fig. 2. The value $N_{\sigma}$ is a ratio of the CNT intrinsic conductivity to the effective LCS conductivity [see Eq. (25)]. As shown in Table I, the conductance $G_{d}$ decreases as the number of extracted atoms increases, and the value $N_{\sigma}$ varies over a wide range reaching 8300 at 60 extracted carbon atoms. Similar low conductance, $0.032 e^{2} /(\pi \hbar)$ and $0.0032 e^{2} /(\pi \hbar)$, has been found in an experiment for a molecular intertube junction with conducting molecules [19]. Let us note that a significant decrease in the transmission coefficient near the Fermi level for single-walled nicked CNTs was also reported in Ref. [17]. Thus, in the present section we estimated the conductance of a few LCSs that may exist in the CNTs.

\section{NUMERICAL RESULTS AND DISCUSSION}

Let us apply the theoretical model developed in Sec. II to calculate the polarizability of the metallic "armchair" $(5,5)$ single-walled CNT with a length of $1 \mu \mathrm{m}$ and a diameter of $0.68 \mathrm{~nm}$. The CNT contains a single LCS, which has a length of $5 \mathrm{~nm}$ and is located $300 \mathrm{~nm}$ away from the left edge of the CNT, i.e., $d=5 \mathrm{~nm}$ and $r=300 \mathrm{~nm}$ in Fig. 3. The electron relaxation time of $50 \mathrm{fs}$ is assumed [34] to calculate the CNT conductivity $\sigma_{c n}$ using Eq. (7). The effective axial surface conductance of the LCS is determined by $N_{\sigma}$ introduced in Sec. III. Below we shall apply the abbreviation "p-CNT" to indicate the CNT without the LCS. If LCS conductance is zero, the d-CNT will be called a cut CNT (c-CNT). Figure 3 shows schematically these CNTs. Let us note that $N_{\sigma}=1$, $N_{\sigma}>1$, and $N_{\sigma}=\infty$ correspond to the p-, d-, and c-CNTs, respectively. The amplitude of the applied electric field is assumed to be $3 \times 10^{4} \mathrm{~V} / \mathrm{m}$.

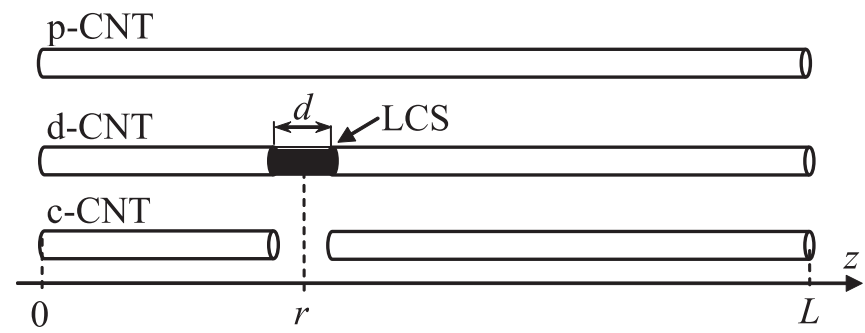

FIG. 3. A schematic depiction of the p-, d-, and c-CNTs. The dark section indicates the LCS with length $d$, and it is located at the distance $r$ away from the left edge of the CNT.

Figure 4 presents the spectral dependences of the real and imaginary parts of the polarizability $\alpha_{z z}$ in the range $1-10^{4}$ $\mathrm{GHz}$ for the d-CNTs at different values $N_{\sigma}$. For the p-CNT $\left(N_{\sigma}=1\right)$, the spectrum of $\operatorname{Im}\left(\alpha_{z z}\right)$ has a terahertz peak at a frequency of $2 \mathrm{THz}$, which is associated with the localized plasmon resonance in the CNTs $[14,34]$. This peak shifts to higher frequencies as the tube length decreases. There is a quasistatic regime of CNT interaction with the electromagnetic field at frequencies below the localized plasmon resonance. In this range, the value of $\operatorname{Re}\left(\alpha_{z z}\right)$ is the same as in the electrostatic case at $f \rightarrow 0$, and the value of $\operatorname{Im}\left(\alpha_{z z}\right)$ decreases with frequency. Due to the strong depolarizing field, the total axial field in the CNT is much lower than the axial component of the incident field.

As shown in Fig. 4, the polarizability spectra of the d-CNT at $N_{\sigma}=20$ and the p-CNT are very similar. There are three peaks for the d-CNTs at $N_{\sigma} \geqslant 200$ in Fig. 4(b). Two of them are at 3.5 and $8 \mathrm{THz}$; they occur due to the localized plasmon resonance in the right and left CNT sections separated by the LCS. The third one is a very broad gigahertz peak; its central frequency $f_{g}$ decreases as $N_{\sigma}$ increases: $f_{g}$ is 10 and $100 \mathrm{GHz}$ for $N_{\sigma}=2 \times 10^{3}$ and $2 \times 10^{4}$, respectively.

To explain the influence of the LCS on the d-CNT polarizability spectrum, we shall consider the field, charge, and current density distributions as well as dissipated power in the d-CNT at $N_{\sigma}=800$. This value of $N_{\sigma}$ was chosen

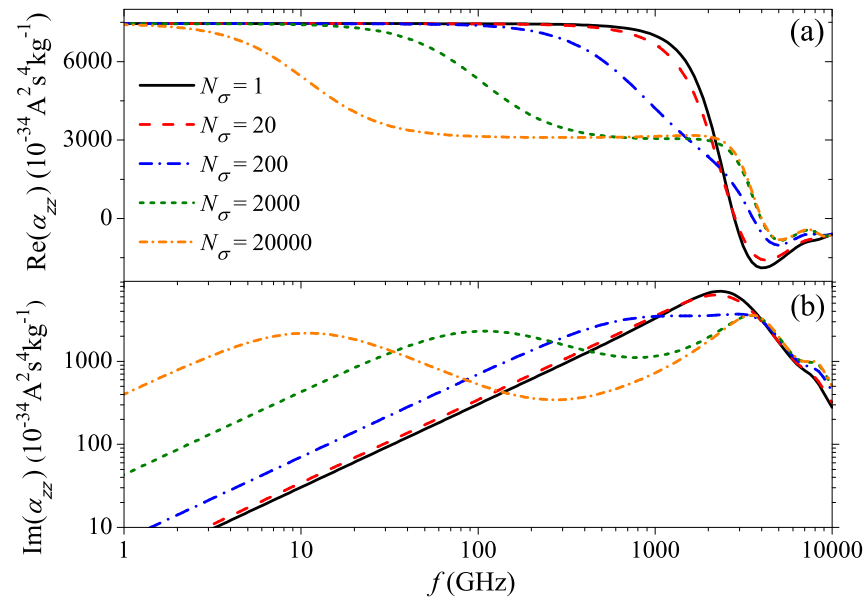

FIG. 4. Frequency dependence of the (a) real and (b) imaginary parts of the d-CNT polarizability for different LCS conductivities $N_{\sigma} \in\left\{1,20,2 \times 10^{2}, 2 \times 10^{3}, 2 \times 10^{4}\right\}$. 


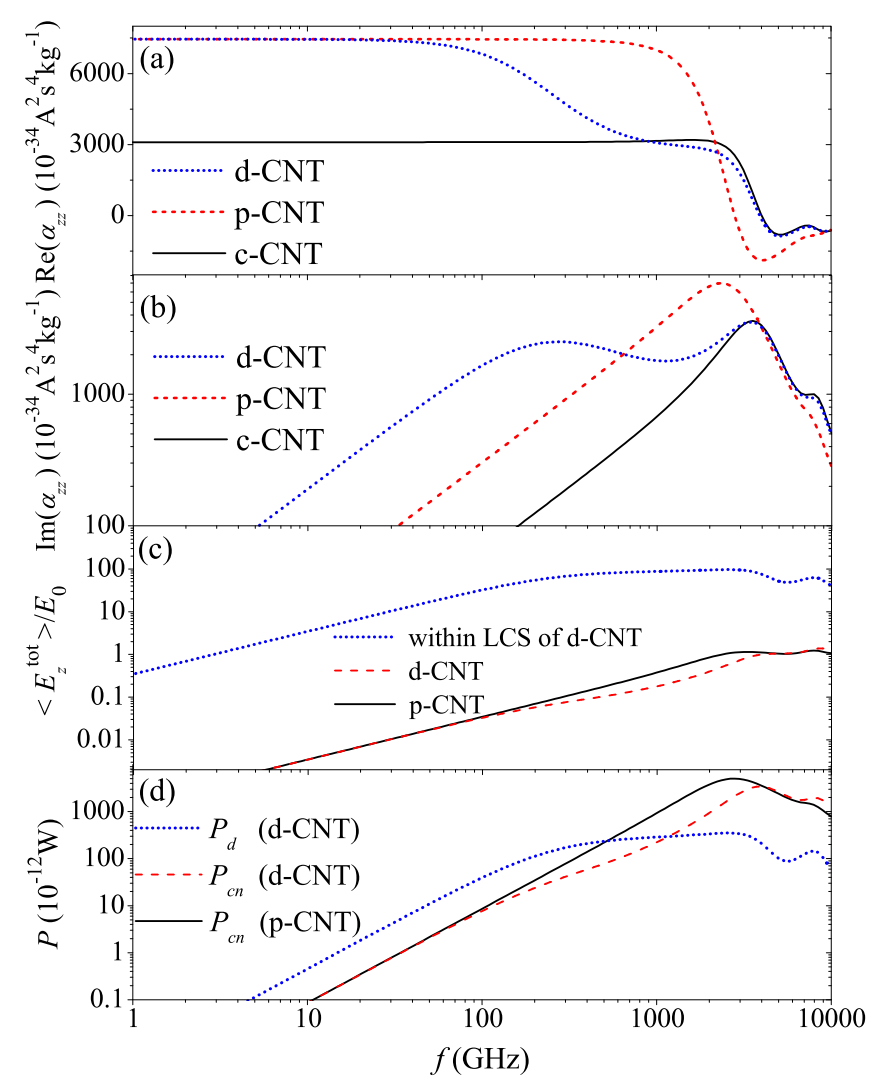

FIG. 5. Frequency dependences of the (a) real and (b) imaginary parts of the polarizability of the d-CNT $\left(N_{\sigma}=800\right)$, c-CNT, and p-CNT. Frequency dependences of (c) normalized average electric field and (d) dissipated power in the p-CNT (solid line) as well as dissipated powers $P_{d}$ (dotted line) and $P_{c n}$ (dashed line) in the d-CNT.

for convenience to compare the current and charge-density distribution over the d-, p-, and c-CNTs on the same scale.

Figures 5(a) and 5(b) show the polarizability spectra for the $\mathrm{p}-, \mathrm{c}-$, and d-CNTs. The frequency dependence of the average electric field and dissipated power in the p-CNT and in the d-CNT (within and outside the LCS) are shown in Figs. 5(c) and 5(d), respectively. In Figs. 5(a) and 5(b), we observe the following:

(i) A regime of the high-transparency LCS $(f<40 \mathrm{GHz})$, where the real part of the polarizability for the d-CNT coincides with that for the p-CNTs.

(ii) A regime of the medium-transparency LCS $(40 \mathrm{GHz}<$ $f<2 \mathrm{THz}$ ), where the polarizabilities of the $\mathrm{d}-, \mathrm{c}-$, and $\mathrm{p}-$ CNTs are different from one another.

(iii) A regime of the low-transparency LCS $(f<2 \mathrm{THz})$, where the polarizabilities of the $\mathrm{d}$ - and c-CNTs almost coincide with each other.

Let us consider each regime in detail.

\section{A. Regime of the high-transparency LCS}

Since the external field oscillates quite slowly, the charge carriers have enough time to pass through the LCS. As a result, the value of $\operatorname{Re}\left(\alpha_{z z}\right)$ is the same for the d-and p-CNTs.

Figure 6 demonstrates the surface charge and current density distributions on the $\mathrm{p}$ - and d-CNTs when the amplitude
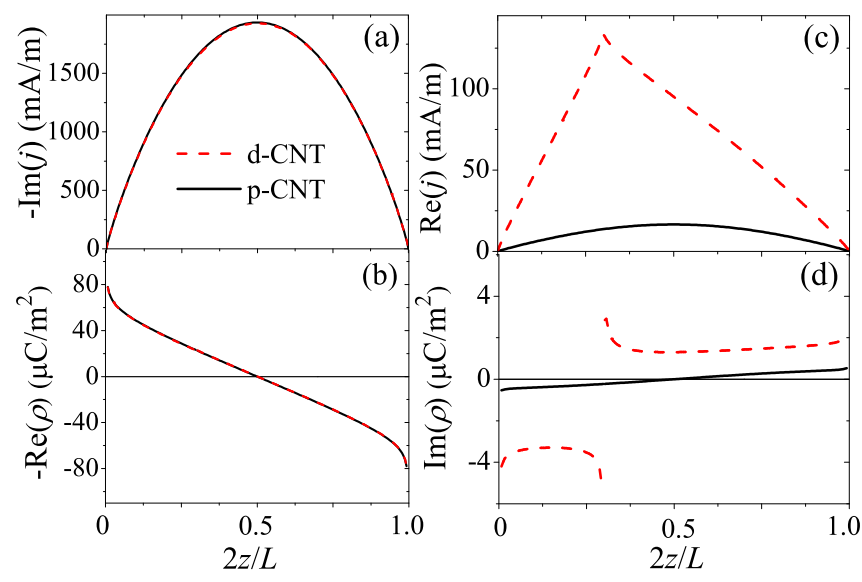

FIG. 6. (a), (c) The surface current and (b), (d) charge-density distribution over the d-CNT $\left(N_{\sigma}=800\right)$ and the p-CNT at a frequency of $20 \mathrm{GHz}$.

of the incident field is real and the frequency is $10 \mathrm{GHz}$. One can see that $\operatorname{Im}(j) \gg \operatorname{Re}(j)$ and $\operatorname{Re}(\rho) \gg \operatorname{Im}(\rho)$, and the values of $\operatorname{Im}(j)$ and $\operatorname{Re}(\rho)$ coincide for the p- and d-CNTs everywhere except in the LCS and its near neighborhood. Due to the charge accumulated in the vicinity of the LCS, the effective field within the LCS is much higher than the average effective field outside the LCS [see Fig. 5(c)]. As a result, the current density within the LCS is the same as it would be in the CNT without the LCS. As the LCS conductance is very low, the dissipated power $P_{d}$ is higher than the power $P_{c n}$ in the d-CNT [Fig. 5(d)]. Thus, the high-energy dissipation near the LCS at $f<800 \mathrm{GHz}$ leads to the higher imaginary part of the polarizability of the d-CNT in comparison to that of the p-CNT [see Fig. 5(b)].

\section{B. Regime of the medium-transparency LCS}

In this regime, the LCS partially hinders electric charge flow. The higher the frequency, the less charge can pass across the LCS during the period of the field oscillations. Then the current density on the LCS decreases as the frequency increases. As a result, the value of $\operatorname{Re}\left(\alpha_{z z}\right)$ decreases and the value of $\operatorname{Im}\left(\alpha_{z z}\right)$ has a peak at $f_{g}=10 \mathrm{GHz}$ [Fig. 5(c)]. The peak is a result of two physical phenomena in the d-CNT: (i) a formation of the depolarizing field in a finite length d-CNT, and (ii) a limitation on the charge propagation through the LCS. Therefore, the frequency $f_{g}$ depends on the CNT length as well as on the conductance of the LCS and its position in the d-CNTs.

Figure 7 shows the current and charge distributions on the $\mathrm{d}-$ and p-CNTs at a frequency of $300 \mathrm{GHz}$. One can see from Fig. 7 that the LCS modifies the magnitude and phase of the current and charge on the d-CNT in comparison to those on the p-CNT.

The surface charge and field distributions over the d-CNT are schematically illustrated in Fig. 8, when the incident field $\mathbf{E}_{0}$ is polarized along the nanotube axis. Due to the edge effects, a large surface density of electric charge $\rho_{2}$ accumulates near the d-CNT edges. This charge induces a depolarizing field $\mathbf{E}_{d}$ with magnitude $\left|\mathbf{E}_{d}\right| \approx\left|\mathbf{E}_{0}\right|$ resulting in a very small total field 

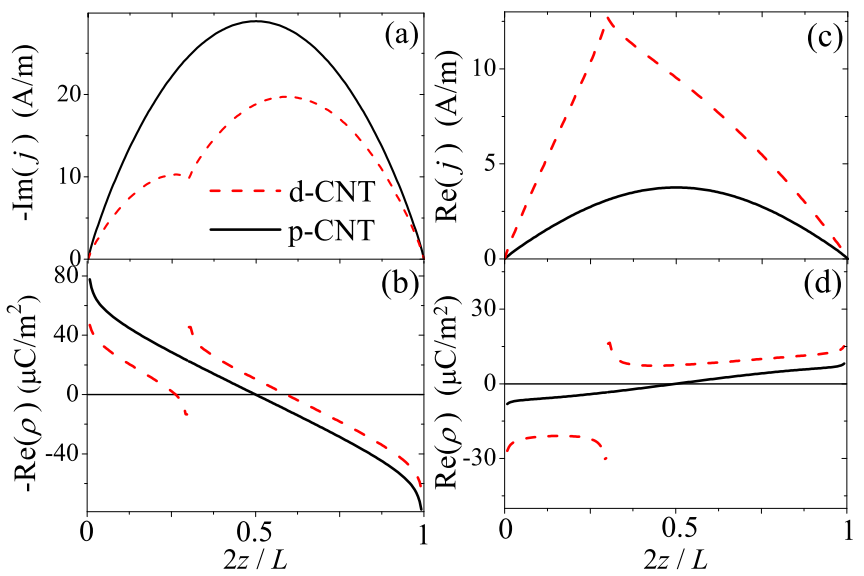

FIG. 7. Same as in Fig. 6, but at a frequency of $300 \mathrm{GHz}$.

$\left|\mathbf{E}_{t}\right|=\left|\mathbf{E}_{0}+\mathbf{E}_{d}\right| \ll\left|\mathbf{E}_{0}\right|$. The charges accumulated on both sides of the LCS with the density $\rho_{1}$ induce a high electric field $\mathbf{E}_{\mathrm{LCS}}$ within the LCS, i.e., $\mathbf{E}_{\mathrm{LCS}} \gg\left|\mathbf{E}_{0}\right|$. As shown in Fig. 5(d), the total axial field outside the LCS is about 1000 times smaller than the field within the LCS. Thus, the near-field enhancement effect occurs within the LCS, while the screening effect takes place outside the LCS.

\section{Regime of the low-transparency LCS}

In this regime, a very small charge passes through the LCS during the period of the field oscillations, i.e., the LCS divides the CNT into two parts interacting with one another only via the electromagnetic field.

Figure 9 shows the charge and current distributions over the d-and c-CNTs at a frequency of $5 \mathrm{THz}$. One can see that both the surface charge and current on the d-CNT coincide with those on the c-CNT. Consequently, the polarizability of the d-CNTs is almost the same as that for the c-CNT at $f>2$ THz [see Figs. 5(a) and 5(b)].

Figure 10 demonstrates the polarizability spectra of the pand d-CNTs with different LCS locations. As shown in Fig. 10, the difference between the spectra of the p- and d-CNTs is (i) the smallest in the case of the LSC located in the vicinity of the CNT edges, and (ii) the highest for the LCS located in the center of the d-CNT. The gigahertz peak frequency $f_{g}$ is the smallest for the case of the LSC located in the center of the d-CNT, and it increases as the LCS shifts toward the edge of the tube. The closer the LCS is to the tube center, the more charges pass through it during one period of the

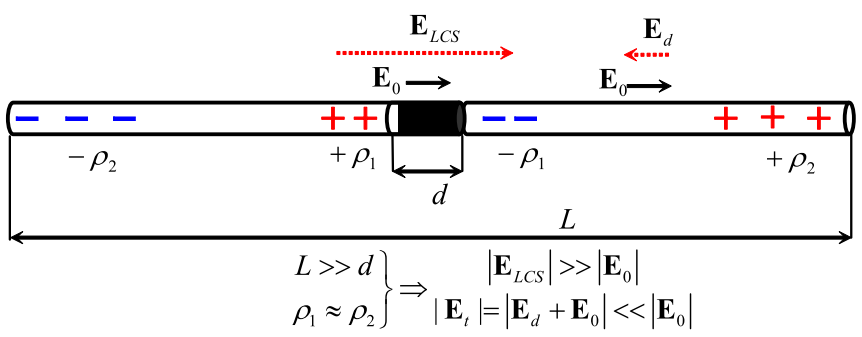

FIG. 8. A schematic depiction of the charge and electric-field distributions on the d-CNT.
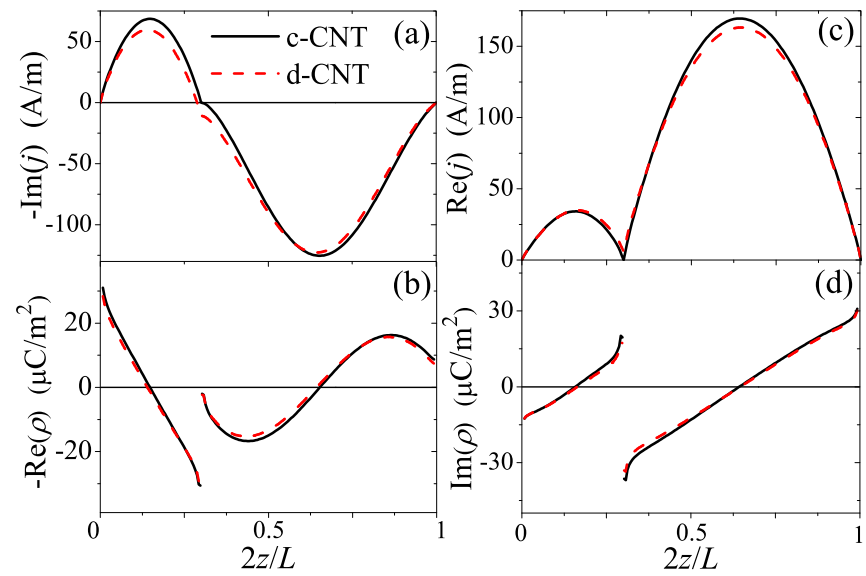

FIG. 9. Same as in Fig. 6, but at a frequency of $5 \mathrm{THz}$.

field oscillation. As a result, the dissipated power $P_{d}$ and, consequently, the imaginary part of the d-CNT polarizability are maximal in the low-frequency range for the case of the LCS located in the tube center.

Figure 11 demonstrates the polarizability spectra of the d-CNTs with different lengths at the same relative position $r / L$ and length $d$ of the LCS. As shown in Fig. 11(b), the frequencies of both the terahertz and gigahertz peaks are lower for the longer nanotube. The longer the d-CNT, the more time is needed for charges to be redistributed following the oscillations of the external field. Moreover, the longer the tubes, the more charge should cross the LCS during the period of field oscillations. This explains the length dependence of the gigahertz peak.

\section{THE POLARIZABILITY OF THE d-CNT WITH MANY LCSs}

In the present section, we apply the theoretical approach developed in Sec. II to calculate the polarizability of a metallic $(5,5)$ CNT with $n$ identical LSCs. Let a tube length be $L=$ $1 \mu \mathrm{m}$, and let the LSCs be located equidistant from each other.

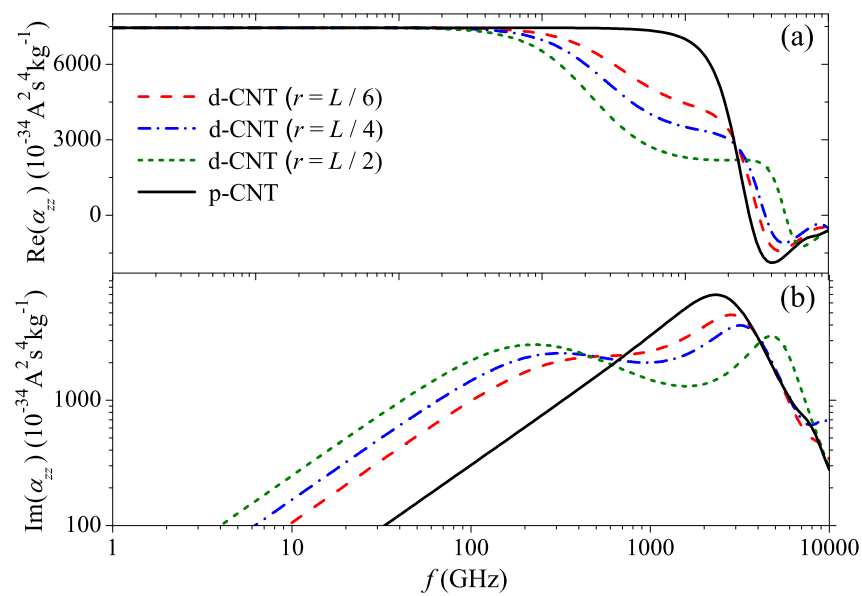

FIG. 10. Frequency dependence of the (a) real and (b) imaginary parts of the p- and d-CNT polarizability at different LSC locations $r \in\{L / 6, L / 4, L / 2\}, N_{\sigma}=800$. 


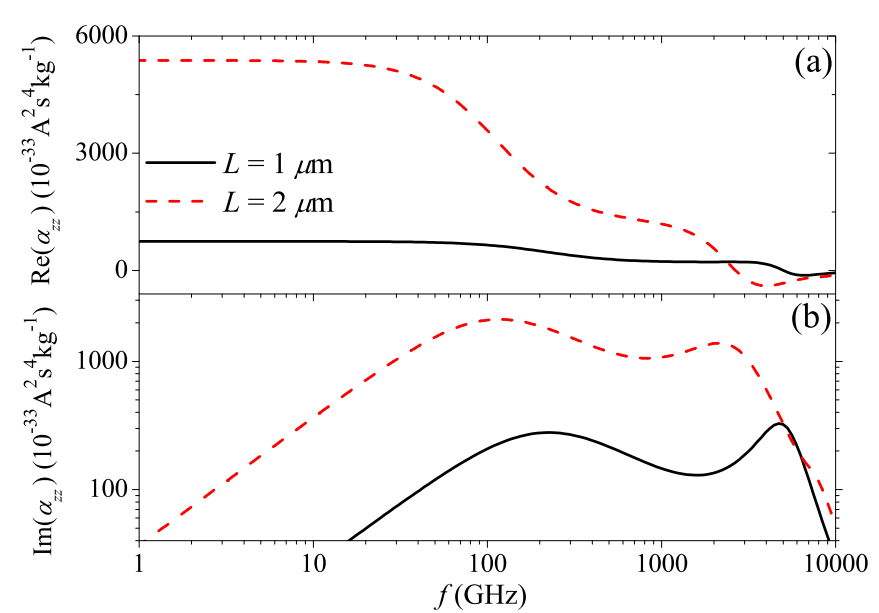

FIG. 11. Frequency dependence of the (a) real and (b) imaginary parts of the d-CNT polarizability for different CNT lengths: $L=1$ and $2 \mu \mathrm{m}$. The LCS is located in the center of the d-CNT and $N_{\sigma}=$ 800 .

Each LCS has a length of $5 \mathrm{~nm}$ and a conductance determined by $N_{\sigma}=800$.

Figure 12 shows the polarizability spectra for the p-CNT and d-CNTs with different numbers, $n$, of the LCSs. As shown in Fig. 12(b), all the spectra $\operatorname{Im}\left[\alpha_{z z}(\omega)\right]$ have one gigahertz peak, whose frequency decreases as the value of $n$ increases. Due to the strong energy dissipation near the LCSs, the imaginary part of the polarizability is much higher for the dCNTs than for the p-CNT at low frequencies $(f<500 \mathrm{GHz})$. Thus, one can increase the microwave energy absorption in an individual d-CNT by adding the LCSs. In the high-frequency range $f \in(0.6,10) \mathrm{THz}$, both the real and imaginary parts of the polarizability are higher for the p-CNT than for the d-CNT.

A d-CNT with a large number of LCSs can be considered as the p-CNT with diminished effective axial conductivity. To demonstrate this, we calculated the polarizability spectrum

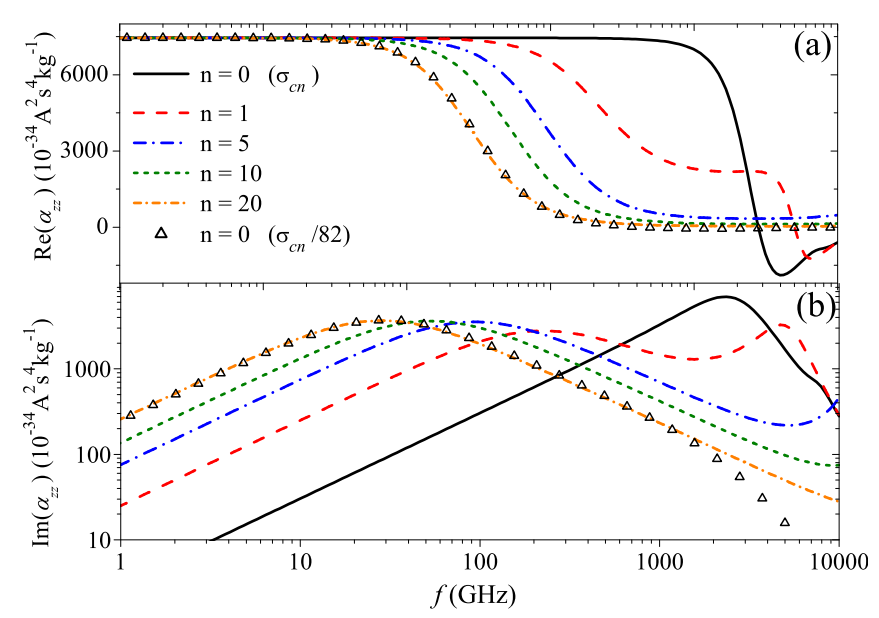

FIG. 12. Frequency dependence of the (a) real and (b) imaginary parts of the polarizability for the d-CNTs with various numbers of identical LCSs $n \in\{1,5,10,20\}$, and for the p-CNTs $(n=0)$ with different axial CNT conductivities $\sigma_{c n}$ and $\sigma_{c n} / 82$, where $\sigma_{c n}$ is determined by (7). of the p-CNT with diminished conductivity $\sigma_{c n} / 82$, and we found good agreement of this spectrum with that of the d-CNT with $n=20$ (see Fig. 12). It is worth noting that the total conductance of the d-CNT with 20 LCSs and the conductance of the p-CNT with diminished conductivity are approximately the same. Note that the same behavior is observed for the system of two barriers separated by a region with strong electron dephasing processes: the resistance of the system is equal to the sum of the intrinsic resistances of these two barriers [46].

Our calculations also show a small difference between the spectra of the d-CNTs at both ordered and random distributions of the LCSs in the nanotube at low frequencies $(1<f<100 \mathrm{GHz})$ if the number of LCSs is large $(n>10)$. Thus, to calculate the polarizability of the d-CNT with many ordered or randomly located LCSs, one can use the simpler model of the p-CNT with the conductance equal to the effective conductance of the d-CNT.

\section{TUNABILITY OF THE d-CNT POLARIZABILITY VIA DRIVING dc VOLTAGE AND SOME POTENTIAL APPLICATIONS}

In this section, we consider the possibility of tuning the LCS ac conductance by the applied dc voltage. Toward that end, we need to apply the two-terminal Landauer-Büttiker formalism [49] for the case of different electrochemical potentials on the left and right sides of the LCS, denoted below by indexes $\alpha$ and $\beta(\alpha, \beta=1,2)$ and considered as electron reservoirs. The generalization can be done correctly taking into account the self-consistent screening effect [49]. In this case, the ac current depends on both the applied external potential $V_{\beta}$ and the oscillating internal potential $U$ at the center of the LCS produced by the displacement capacitive currents on both sides of the LCS. As a result, the total current on the $\alpha$ side of the LCS is

$$
I_{\alpha}=\sum_{\beta=1,2} g_{\alpha \beta} V_{\beta}+\tilde{g}_{\alpha} U
$$

where

$$
\tilde{g}_{\alpha}=-\sum_{\beta=1,2} g_{\alpha \beta}
$$

and

$$
g_{\alpha \beta}=-\frac{e^{2}}{\pi \hbar} \int T_{\alpha \beta}(E) \frac{\partial f_{\beta}}{\partial E} d E .
$$

Here $f_{\beta}(E)=f\left(E-\mu_{\beta}\right)$ is the electron Fermi function and $\mu_{\beta}$ is electrochemical potential on the $\beta$ side of the LCS, $\mu_{1}-\mu_{2}=e V_{\mathrm{dc}}, V_{\mathrm{dc}}$ is the dc voltage across the LCS. The internal potential is [49]

$$
U=\frac{\sum_{\alpha, \beta=1,2} g_{\alpha \beta} V_{\beta}+i \omega \sum_{\beta=1,2} C_{\beta} V_{\beta}}{\sum_{\alpha, \beta=1,2} g_{\alpha \beta}+i \omega \sum_{\beta=1,2} C_{\beta}},
$$

where $C_{\beta}$ is the capacitance between the point with potential $U$ and the $\beta$ side of the LCS. Using the sum rules [49], we find the current through the LCS,

$$
I_{d}=g_{11} V_{1}-g_{22} V_{2}-\frac{g_{11}-g_{22}}{C_{1}+C_{2}}\left(C_{1} V_{1}+C_{2} V_{2}\right) .
$$


Due to the second term determined by the self-consistent screening, Eq. (38) is invariant under an equal shift of both potentials. For symmetric excitation, we have $V_{1,2}= \pm V_{d} / 2$, which corresponds to the LCS conductance

$$
G_{d}=\frac{1}{2}\left[g_{11}+g_{22}-\left(g_{11}-g_{22}\right) \frac{C_{1}-C_{2}}{C_{1}+C_{2}}\right] .
$$

For the symmetric configuration of the LCS geometry $C_{1}=$ $C_{2}$, we find

$$
G_{d}=\frac{e^{2}}{\pi \hbar}\left[T\left(E_{F}-e V_{\mathrm{dc}} / 2\right)+T\left(E_{F}+e V_{\mathrm{dc}} / 2\right)\right] .
$$

One can see from (40) that the dc voltage $V_{\mathrm{dc}}$ applied to the mesoscopic system can lead to the variation of its ac conductance $G_{d}(\omega)$. This shows the possibility of electrically tuning the polarizability $\alpha_{z z}(\omega)$ given by (30). This result paves the way for efficient implementation of controllable nanoantennas and lumped elements of nanocircuits in the gigahertz and terahertz ranges.

Another important application is related to the well-known ability of the electrons to produce a shot noise by pushing them through a quantum point contact with a driving dc field [64-67]. As noted in Ref. [67], such a noise may be considered as a source of quantum low-frequency radiation, which is difficult to generate using any other technique. In particular, the quantum point contact fabricated through gates, constricting the electron flow in a two-dimensional electron gas, is able to emit plasmons that may be transformed into quanta of an EM field in the LC circuits or transmission lines. The wavelength of the plasmons involved in the experiment [66] is even larger than the sample's size. In this case, the charge fluctuations generated at the quantum point contact are directly coupled into the transmission lines, whereas the direct photonic emission is a problem. For an effective photonic emission, the plasmon wave should be transformed into free three-dimensional photons. To achieve this, one needs to match the impedance between the mesoscopic setup (with an electric circuit environment) and free space. Nanoantennas are an efficient tool to facilitate such photon emission [7-9].

Thus, we propose the system considered above as a promising candidate for quantum antenna feed by shot noise for emitting the quanta of gigahertz and terahertz radiation. The statistics of this radiation can be controlled from bunching to antibunching by varying the dc voltage [67]. The improvement of the radiation parameters can be done by means of classical antenna engineering [15]. For example, it might be an antenna array with one dipole serving as an exciter, and all others acting as parasitic directors (Yagi-Uda antennas [15]). Such antennas may be implemented based on various types of nanostructures, for example a circular loop with a mesoscopic setup, a helix, etc.

\section{CONCLUSION AND OUTLOOK}

To summarize, we have developed the integral equation technique for the problem of electromagnetic scattering by a finite-length nanowire with a number of embedded mesoscopic objects. The theory is based on combining the integral equations of the classical antenna theory (Hallén-type equations) with the quantum transport formalism $[47,49]$. The generalized equations comprise the integral operators of wire antennas and algebraic terms responsible for the mesoscopic systems. Such equations are solvable numerically for a wide range of frequencies, as well as constitutive and size parameters of the systems.

The developed technique was adapted to the problem of electromagnetic scattering by stand-alone finite-length CNT with a short LCS. The structure under consideration is promising for the realization of nanoantennas in the terahertz frequency range. Electrical transport through the LCS has been described by the Landauer approach in terms of the electron transmission coefficient $T$, while effective resistive sheet impedance boundary conditions have been used to describe the current induced on the CNT surface; the Drude model has been used to calculate CNT axial conductivity outside the LCS. Based on DFT, ab initio calculations have been carried out to estimate the multichannel electron transmission coefficient of a few particular LCSs constructed by extraction of carbon atoms in the CNT.

The modified Hallén integral equation for the d-CNT was formulated and solved numerically. The electromagnetic parameters of the d-CNT, such as (i) the surface current density, (ii) the surface charge density, (iii) the tensor of the polarizability, and (iv) the dissipative power, have been calculated in a wide frequency range $(1-10000 \mathrm{GHz})$. It has been shown that the electromagnetic characteristics of the d-CNTs are different from those for the p-CNTs. The calculated polarizability spectra of the d-CNT were shown to have three peaks: two terahertz peaks, which are due to the localized plasmon resonances in the CNT sections separated by the LCS, and one gigahertz peak, due to a combination of the finite-length effect in the d-CNT and a limitation on the charge crossing the LCS. The frequency of the gigahertz peak depends on the conductance of the LCS and its position in the d-CNT; it separates the regimes of low and high transparency for charge transfer through the LCS. The polarizability spectrum for the d-CNT comprising many ordered identical LCSs was shown to have one gigahertz peak. The frequency of the peak decreases as the number of LCSs increases. Energy dissipation in the $\mathrm{d}-\mathrm{CNT}$ is higher than that in the $\mathrm{p}-\mathrm{CNT}$ in the gigahertz range, whereas the opposite is true in the terahertz range. The gigahertz response of the d-CNT is very similar to the response of the p-CNT with the same total conductance.

The predicted polarizability peaks are promising from a practical point of view as a framework for enhancement of the quantum antenna efficiency in the terahertz and gigahertz ranges. The current fluctuations in the LCS and, consequently, the probability of the bunched photon emission can be enhanced via an increase of the CNT polarizability.

\section{ACKNOWLEDGMENTS}

This research was partially supported by the Belarusian Republican Foundation for Fundamental Research (BRFFR) under project F15CO-016, the EU FP7 under projects FP7612285 CANTOR, PIRSES-GA-2012-318617, and by the H2020-MSCA-RISE-2014 project 644076 CoExAN. CPU time was granted by the CINECA supercomputer center (ISCRA C HP10CQXAWA). 
[1] G. B. Lesovik and I. A. Sadovskyy, Phys. Usp. 54, 1007 (2011).

[2] M. A. Kastner, Phys. Today 46(1), 24 (1993).

[3] H. v. Houten and C. Beenakker, Phys. Today 49(7), 22 (1996).

[4] R. J. Nichols and S. J. Higgins, Nat. Mater. 14, 465 (2015).

[5] S. Reich, C. Thomsen, and J. Maultzsch, Carbon Nanotubes. Basic Concepts and Physical Properties (Wiley-VCH, Berlin, 2004).

[6] R. Landauer, IBM J. Res. Dev. 1, 223 (1957).

[7] P. Biagioni, J.-S. Huang, and B. Hecht, Rep. Prog. Phys. 75, 024402 (2012).

[8] L. Novotny and N. van Hulst, Nat. Photon. 5, 83 (2011).

[9] A. Alú and N. Engheta, Phys. Rev. Lett. 101, 043901 (2008).

[10] A. J. White, M. Sukharev, and M. Galperin, Phys. Rev. B 86, 205324 (2012).

[11] L. Meng, Z. Yin, C. Yam, S. Koo, Q. Chen, N. Wong, and G. Chen, J. Chem. Phys. 139, 244111 (2013).

[12] C. Yam, L. Meng, Y. Zhang, and G. Chen, Chem. Soc. Rev. 44, 1763 (2015).

[13] G. Hanson, IEEE Trans. Antennas Propag. 53, 3426 (2005).

[14] G. Y. Slepyan, M. V. Shuba, S. A. Maksimenko, and A. Lakhtakia, Phys. Rev. B 73, 195416 (2006).

[15] C. A. Balanis, Antenna Theory, 3rd ed. (Wiley, New York, 1997).

[16] N. Engheta, A. Salandrino, and A. Alù, Phys. Rev. Lett. 95, 095504 (2005).

[17] W. Ren and J. Wang, Phys. Rev. B 69, 033306 (2004).

[18] C. Dekker, Z. Yao, H. W. C. Postma, and L. Balents, Nature (London) 402, 273 (1999).

[19] X. Guo, Science 311, 356 (2006).

[20] S. J. van der Molen, J. Liao, T. Kudernac, J. S. Agustsson, L. Bernard, M. Calame, B. J. van Wees, B. L. Feringa, and C. Schönenberger, Nano Lett. 9, 76 (2008).

[21] A. J. Kronemeijer, H. B. Akkerman, T. Kudernac, B. J. van Wees, B. L. Feringa, P. W. Blom, and B. de Boer, Adv. Mater. 20, 1467 (2008).

[22] H. Song, Y. Kim, Y. H. Jang, H. Jeong, M. A. Reed, and T. Lee, Nature (London) 462, 1039 (2009).

[23] D. M. Cardamone, C. A. Stafford, and S. Mazumdar, Nano Lett. 6, 2422 (2006).

[24] M. Di Ventra, S. Pantelides, and N. Lang, Appl. Phys. Lett. 76, 3448 (2000).

[25] R. Gutiérrez, G. Fagas, G. Cuniberti, F. Grossmann, R. Schmidt, and K. Richter, Phys. Rev. B 65, 113410 (2002).

[26] B. Q. Xu, X. L. Li, X. Y. Xiao, H. Sakaguchi, and N. J. Tao, Nano Lett. 5, 1491 (2005).

[27] A. Ugawa, A. G. Rinzler, and D. B. Tanner, Phys. Rev. B 60, R11305 (1999).

[28] G. Y. Slepyan, S. A. Maksimenko, A. Lakhtakia, O. Yevtushenko, and A. V. Gusakov, Phys. Rev. B 60, 17136 (1999).

[29] T.-I. Jeon, K.-J. Kim, C. Kang, I. H. Maeng, J.-H. Son, K. H. An, J. Y. Lee, and Y. H. Lee, J. Appl. Phys. 95, 5736 (2004).

[30] P. J. Burke, S. Li, and Z. Yu, IEEE Trans. Nanotechnol. 5, 314 (2006).

[31] M. V. Shuba, S. A. Maksimenko, and A. Lakhtakia, Phys. Rev. B 76, 155407 (2007).

[32] C. Rutherglen, D. Jain, and P. Burke, Appl. Phys. Lett. 93, 083119 (2008).

[33] A. Maffucci, G. Miano, and F. Villone, IEEE Trans. Nanotechnol. 8, 345 (2009).

[34] G. Y. Slepyan, M. V. Shuba, S. A. Maksimenko, C. Thomsen, and A. Lakhtakia, Phys. Rev. B 81, 205423 (2010).
[35] M. V. Shuba, G. Y. Slepyan, S. A. Maksimenko, and G. W. Hanson, J. Appl. Phys. 108, 114302 (2010).

[36] M. V. Shuba, A. V. Melnikov, A. G. Paddubskaya, P. P. Kuzhir, S. A. Maksimenko, and C. Thomsen, Phys. Rev. B 88, 045436 (2013).

[37] R. R. Hartmann, J. Kono, and M. E. Portnoi, Nanotechnology 25, 322001 (2014).

[38] P. J. Burke, IEEE Trans. Nanotechnol. 1, 129 (2002).

[39] P. Kuzhir, A. Paddubskaya, D. Bychanok, A. Nemi-lentsau, M. Shuba, A. Plusch, S. Maksimenko, S. Bellucci, L. Coderoni, F. Micciulla, I. Sacco, G. Rinaldi, J. Macutkevic, D. Seliuta, G. Valusis, and J. Banys, Thin Solid Films 519, 4114 (2011).

[40] M. A. Seo, J. H. Yim, Y. H. Ahn, F. Rotermund, D. S. Kim, S. Lee, and H. Lim, Appl. Phys. Lett. 93, 231905 (2008).

[41] M. V. Shuba, A. G. Paddubskaya, A. O. Plyushch, P. P. Kuzhir, G. Y. Slepyan, S. A. Maksimenko, V. K. Ksenevich, P. Buka, D. Seliuta, I. Kasalynas, J. Macutkevic, G. Valusis, C. Thomsen, and A. Lakhtakia, Phys. Rev. B 85, 165435 (2012).

[42] M. V. Shuba, S. A. Maksimenko, and G. Y. Slepyan, J. Comput. Theor. Nanosci. 6, 2016 (2009).

[43] M. V. Shuba, A. G. Paddubskaya, P. P. Kuzhir, S. A. Maksimenko, G. Valusis, M. Ivanov, J. Banys, V. Ksenevich, and G. W. Hanson, Mater. Res. Express 4, 075033 (2017).

[44] P. Giannozzi, S. Baroni, N. Bonini, M. Calandra, R. Car, C. Cavazzoni, D. Ceresoli, G. L. Chiarotti, M. Cococcioni, I. Dabo, A. D. Corso, S. de Gironcoli, S. Fabris, G. Fratesi, R. Gebauer, U. Gerstmann, C. Gougoussis, A. Kokalj, M. Lazzeri, L. Martin-Samos, N. Marzari, F. Mauri, R. Mazzarello, S. Paolini, A. Pasquarello, L. Paulatto, C. Sbraccia, S. Scandolo, G. Sclauzero, A. P. Seitsonen, A. Smogunov, P. Umari, and R. M. Wentzcovitch, J. Phys.: Condens. Matter 21, 395502 (2009).

[45] M. Büttiker, Y. Imry, R. Landauer, and S. Pinhas, Phys. Rev. B 31, 6207 (1985).

[46] M. D. Ventra, Electrical Transport in Nanoscale Systems (Cambridge University Press, Cambridge, UK, 2008).

[47] A. Kamenev and W. Kohn, Phys. Rev. B 63, 155304 (2001).

[48] Z. Iluz and A. Boag, Opt. Lett. 36, 2773 (2011).

[49] M. H. Pedersen and M. Büttiker, Phys. Rev. B 58, 12993 (1998).

[50] M. Büttiker, J. Phys.: Condens. Matter 5, 9361 (1993).

[51] M. Büttiker, A. Pretre, and H. Thomas, Phys. Rev. Lett. 70, 4114 (1993).

[52] G. B. Lesovik and L. S. Levitov, Phys. Rev. Lett. 72, 538 (1994).

[53] A. A. Odintsov and Y. Tokura, Physica B 284-288, 1752 (2000)

[54] S. Tasaki, K. Maekawa, and T. Yamabe, Phys. Rev. B 57, 9301 (1998).

[55] T. Christen and M. Büttiker, Phys. Rev. Lett. 77, 143 (1996).

[56] A. Forouzmand, H. M. Bernety, and A. B. Yakovlev, Phys. Rev. B 92, 085402 (2015).

[57] L. A. Weinstein, The Theory of Diffraction and the Factorization Method (Golem, New York, 1969).

[58] V. L. Gurevich, Phys. Rev. B 55, 4522 (1997).

[59] L. C. Venema, J. W. G. Wildoer, H. L. J. T. Tuinstra, C. Dekker, A. G. Rinzler, and R. E. Smalley, Appl. Phys. Lett. 71, 2629 (1997).

[60] B. Huang, Z. Li, Z. Liu, G. Zhou, S. Hao, J. Wu, B.-L. Gu, and W. Duan, J. Phys. Chem. C 112, 13442 (2008).

[61] H.-S. Sim, C.-J. Park, and K. J. Chang, Phys. Rev. B 63, 073402 (2001). 
[62] H. J. Choi and J. Ihm, Phys. Rev. B 59, 2267 (1999).

[63] J. P. Perdew, J. A. Chevary, S. H. Vosko, K. A. Jackson, M. R. Pederson, D. J. Singh, and C. Fiolhais, Phys. Rev. B 46, 6671 (1992).

[64] U. Gavish, Y. Levinson, and Y. Imry, Phys. Rev. B 62, R10637(R) (2000).
[65] M. Galperin, A. Nitzan, and M. A. Ratner, Phys. Rev. B 74, 075326 (2006).

[66] E. Zakka-Bajjani, J. Ségala, F. Portier, P. Roche, D. C. Glattli, A. Cavanna, and Y. Jin, Phys. Rev. Lett. 99, 236803 (2007).

[67] A. V. Lebedev, G. B. Lesovik, and G. Blatter, Phys. Rev. B 81, 155421 (2010). 